\title{
Detection of the radiolysis of solid ampicillin by UV - spectroscopy
}

\author{
M. Gibella and B. Tilquin* \\ Université catholique de Louvain, Unité d'Analyse Chimique et Physico-Chimique des Médicaments, \\ Laboratoire de Radiostérilisation, Pharmacie, UCL-7230, avenue Mounier 72, 1200 Brussels, Belgium
}

\begin{abstract}
We propose to detect the use of $\gamma$-rays in order to radiosterilize ampicillin by UV absorption spectroscopy. The UV absorption results are verified by comparison with HPLC-diode array analysis. The sensitivity is excellent $\left(10^{-6} \mathrm{M}\right)$. The wavelengths in the range from 287 to $335 \mathrm{~nm}$ are characteristics of radiolysis related substances. It is possible to use this range of wavelengths to detect the radiolysis of ampicillin with an usual UV spectrophotometer.
\end{abstract}

Keywords. antibiotic, radiosterilization, capillary liquid-liquid chromatography, UV-detection.

\section{Introduction}

Many techniques are adapted to the detection of the radiosterilization of pharmaceutical substances. Among them, Electron Paramagnetic Resonance is the most widely recommanded for the detection of irradiated solids by measuring the esr spectra of trapped radicals (1). Thermoluminescence signals are also modified by irradiation (2). Gas-chromatographic techniques may be used to identify "unique" volatile compounds characteristic of the radiosterilization of solid forms (3). HPLC (high performance liquid chromatography) is also useful for studying final radiolytic products (4), some of which are "unique" and characteristic of radiolysis $(3,4,5)$.

All these techniques are adequate for the control and inspection of irradiated drugs, and contribute to the enforcement of Good Irradiation Practice and to prevent impairment of quality (pharmacopeia's guidelines, off-flavors, ...).

A common problem of the radiosterilization of antibiotics is the yellowing of the powder. However, irradiated antibiotics in solid state at low sterilization dose very often meet the pharmacopeia's specifications. The purpose of this study is to use the colour change as a detector for radiosterilization by using simple UV spectrometry; results from this standard technique will be compared with the HPLC-diode array analysis.

\section{Experimental section}

Anhydrous ampicillin acid (AAA) is from Federa Belgium Ltd.
The HPLC system is the following: capillary column $(300 \mu \mathrm{m} \times 25 \mathrm{~cm})$ packed with lichrospher $60 \mathrm{RP}$-select B (5 $\mu \mathrm{m}, 60 \AA$ porosity), $1 \mu \mathrm{l}$ injector and before the injector, a split system allows a flow rate of $4 \mu \mathrm{min}^{-1}$ inside the capillary column. The mobile phase follows a gradient from a mixture $\mathrm{A}\left(\mathrm{ACN}^{1} /\right.$ Acetate buffer, $\left.\mathrm{pH}=5,10 / 90\right)$ to a mixture $\mathrm{B}$ ( $\mathrm{ACN} /$ Acetate buffer, $\mathrm{pH}=5,40 / 60)$.

Radiolysis with doses ranging from 230 to 33,000 Joule $\mathrm{kg}^{-1}($ Gray $=\mathrm{Gy})$ is applied in a gamma room $\left(640 \mathrm{~Gy} \mathrm{~h}^{-1}\right.$ of ${ }^{60} \mathrm{Co}$ ) at $300 \mathrm{~K}$.

After the irradiation, samples are dissolved in MilliQ water and immediately submitted to analysis.

Beckman System Gold with a diode array detector module 512 and conventional column $(25 \mathrm{~cm} \times 4 \mathrm{~mm})$ LICHROCART MERCK are used to obtain individual spectrum of relevant chromatographic peaks.

The UV spectrometer is a double beam UVIKON 933.

\section{Results}

The chromatograms are presented in figure 1 and figure 2 for unirradiated and irradiated (21,000 Gy) samples respectively. The chromatograms are centered on the related substances after the radiolysis. With increasing radiation doses, typical variations are expressed in figure 3 , new peaks at retention times $\left(t_{R}\right)$ of 20,26 and 28 minutes, increase with the radiation dose, however some peaks already present before the irradiation (as example, $t_{R}=22 \mathrm{~min}$ ) decay with increasing radiation dose. The areas under these peaks seem to approach plateau values for the ghest radiation doses, apart from the peak with retention time 28 minutes, which

*Correspondence and reprints.

Received January, 22, 1999; revised July, 7, 1999; accepted July, 15, 1999.

1 acetonitrile 


\section{Original articles}

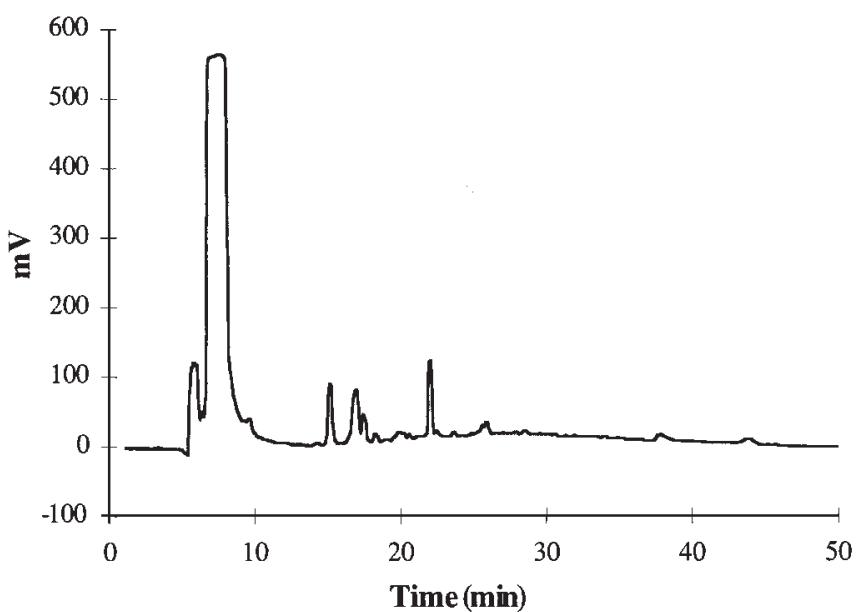

Fig. 1. Chromatogram from nonirrradiated ampicillin (see experimental section).

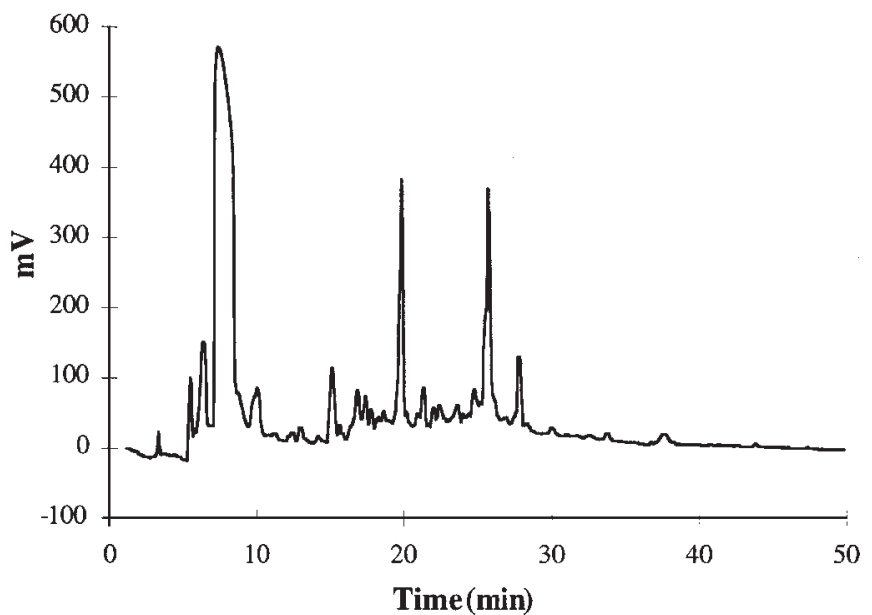

Fig. 2. Chromatogram from ampicillin irradiated to a dose of $21 \mathrm{kGy}$.
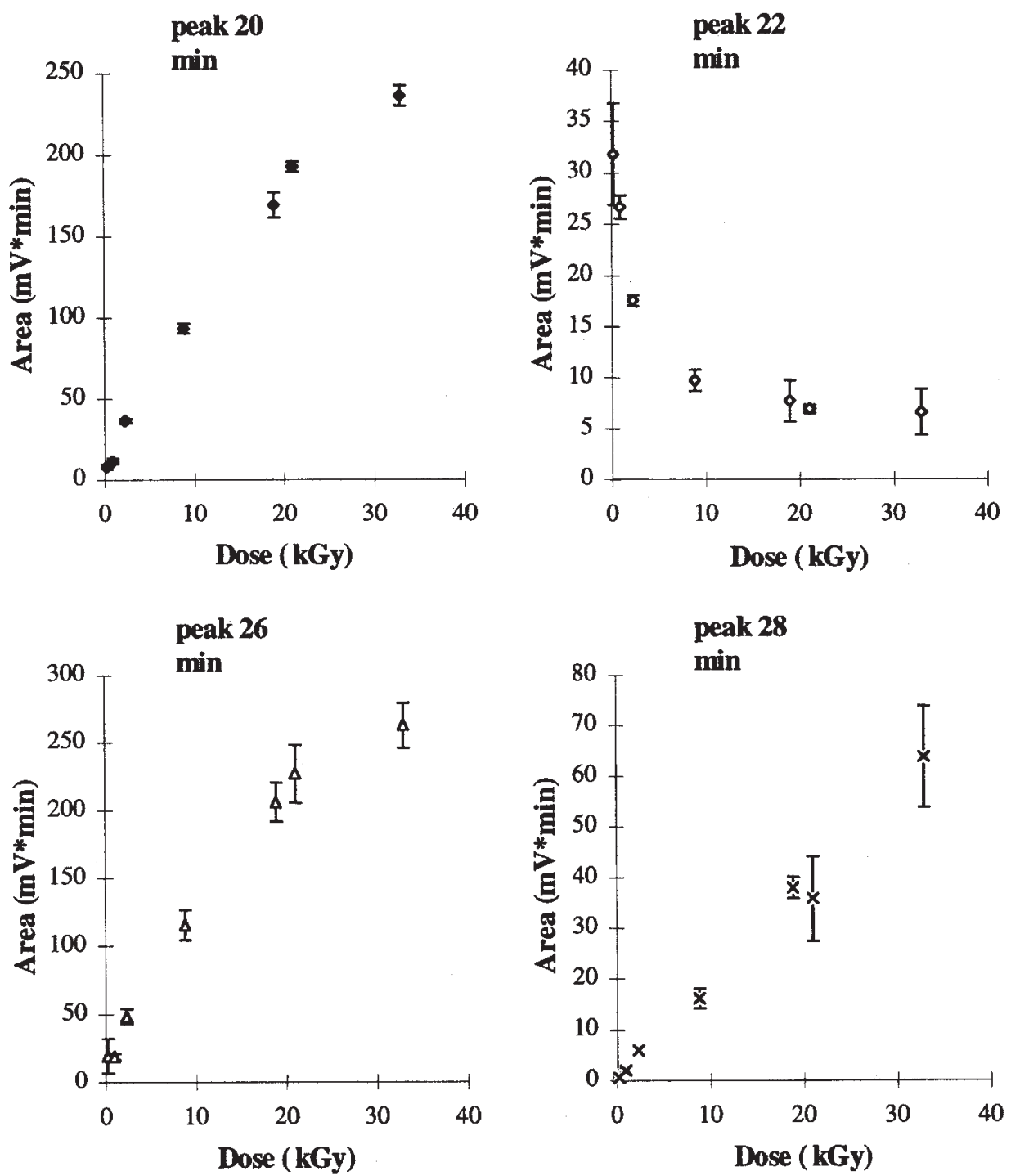

Fig. 3. Some variations of the peak areas (in arbitrary units) as a function of the dose. 


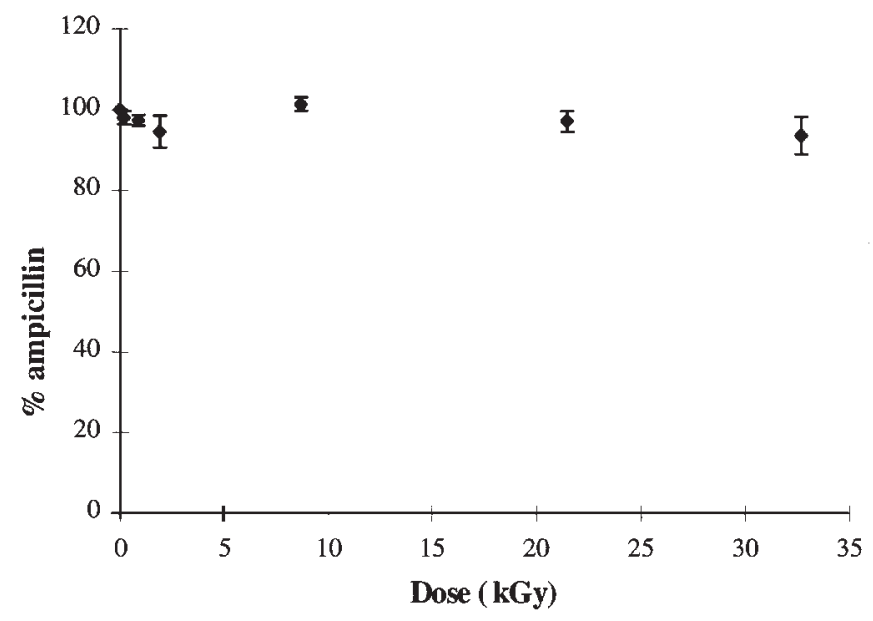

Fig. 4. Potency of the irradiated solid ampicillin as a function of the dose.
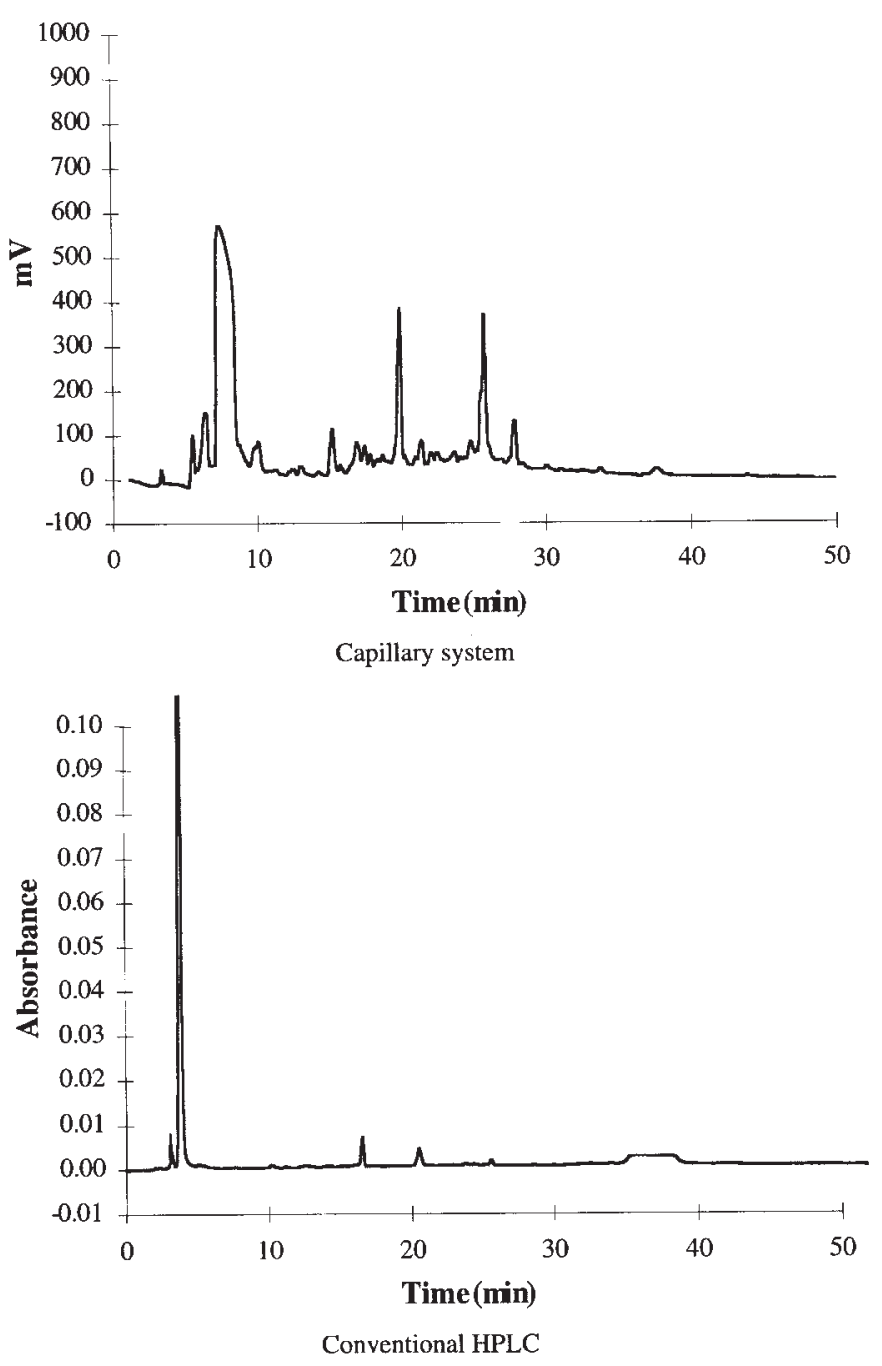

Fig. 5. Comparison of the chromatograms registered with capillary systems (a) and conventional HPLC (b). The dose is of $21 \mathrm{kGy}$.
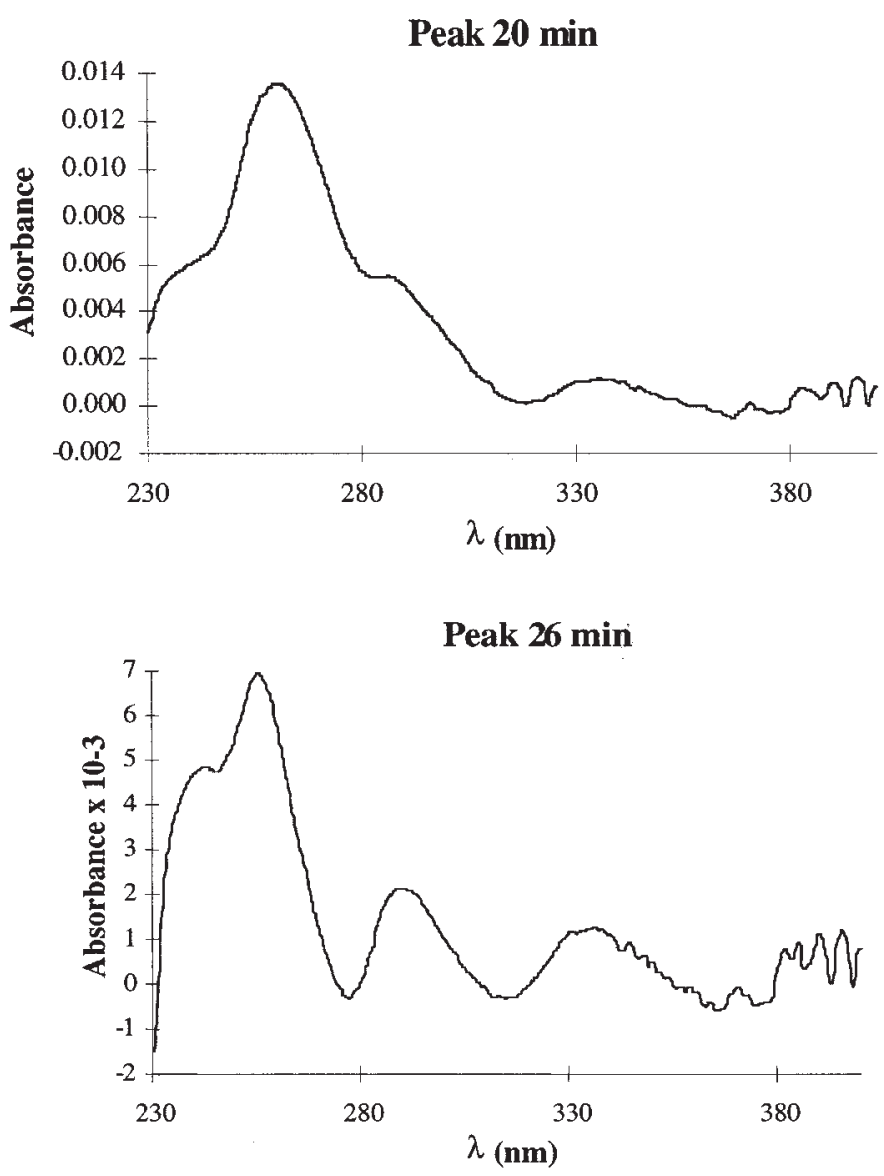

Fig. 6. Diode array spectra from eluted substances, the time of elution is given for capillary system.

appears to be increasing linearly, even at the highest radiation doses studied (40 kGy).

The potency (pharmaceutical power) of ampicillin is not affected by the radiolysis up to $25,000 \mathrm{~Gy}$, the radiation dose arbitrary recommended for irradiation, without studying the relationship dose-sterility $\left(10^{-6}\right.$ SAL: Sterility Assurance Level). The potency, given by analysis of the main chromatographic peak after standardization, is effectively constant as a function of radiation dose (Fig. 4). The maximum loss in percentage is ca. $4 \%$ (relative surface in chromatography) at 25,000 Gy, well within the limit specified by the pharmacopoeia in agreement with the pharmacopoea's specifications (USP XXIII, 10\% loss).

The percentage of total related substance for the same radiation dose is $15 \%$, so the molar absorptivity of the radiolytic products is higher than that of ampicillin with a loss of $4 \%$.

To obtain the characteristic spectrum of each chromatographic peak, we use a diode array detector and a conventional column. The comparison is given in figure 5. Spectra for the peaks after $20 \mathrm{~min}$ and $26 \mathrm{~min}$ in a capillary system are reported in figure 6 . 


\section{Original articles}

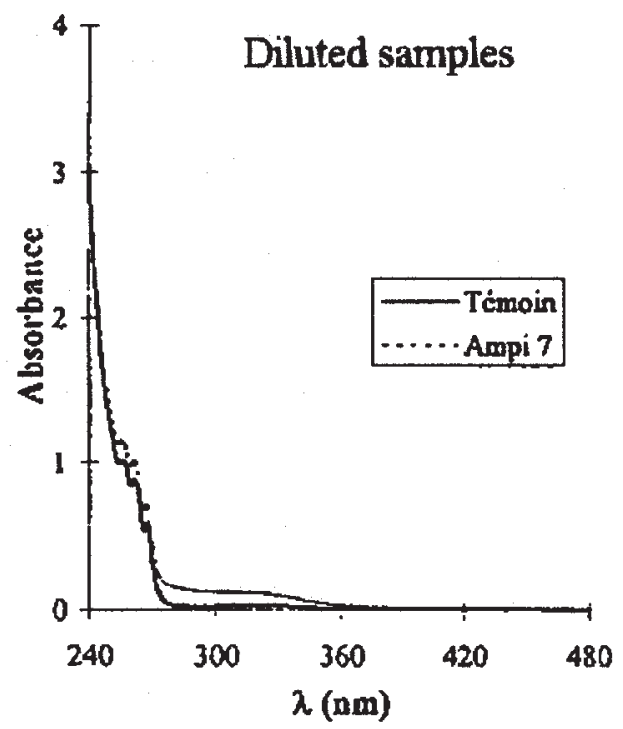

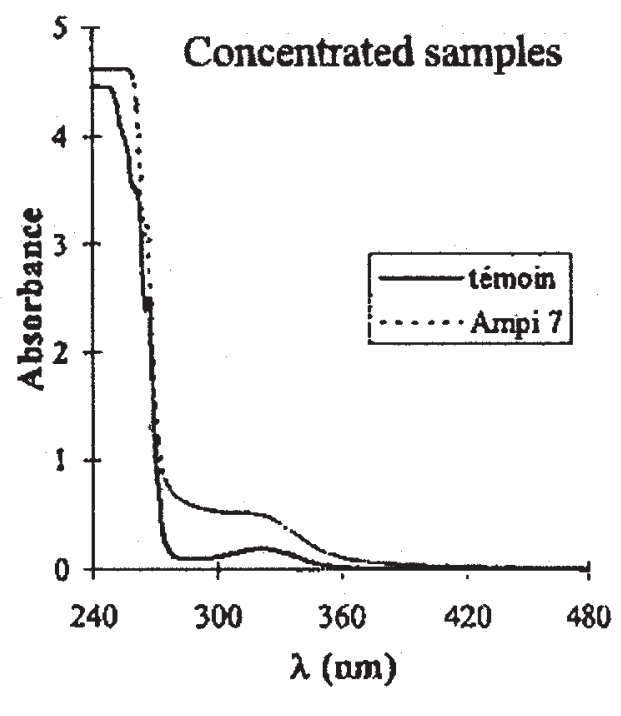

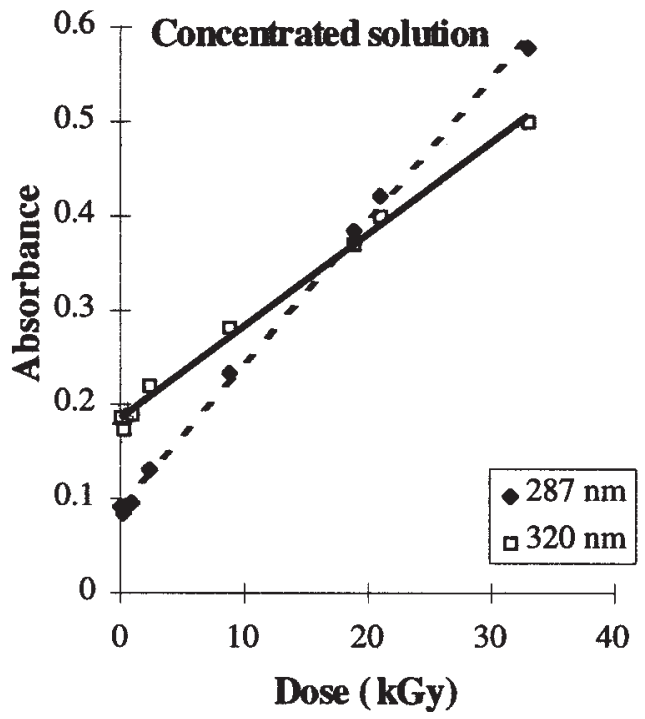

Fig. 7. Usual UV spectra from nonirradiated and irradiated ampicillin.

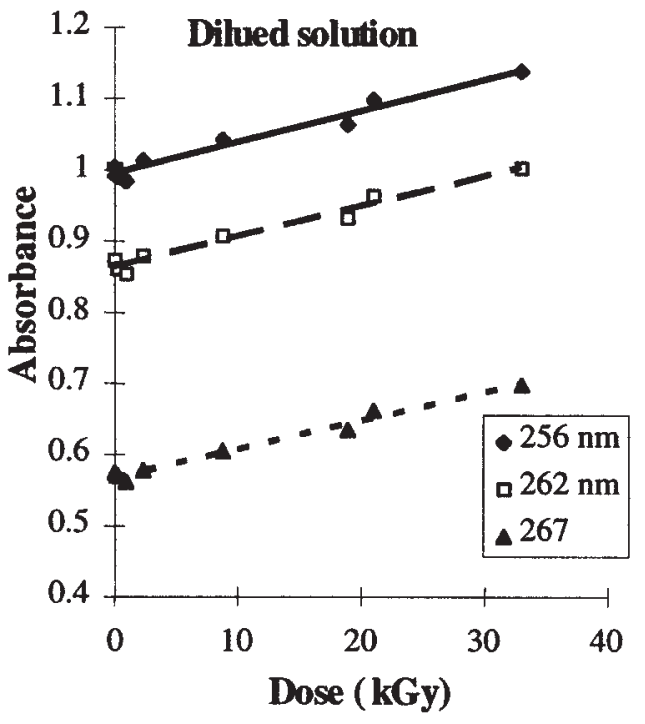

The UV spectra show maxima at 260, 287 and $335 \mathrm{~nm}$; in the same conditions, the UV spectrum of unirradiated ampicillin have maxima at 255, 260 and $270 \mathrm{~nm}$. So wavelengths in the range from 287 to $335 \mathrm{~nm}$ are characteristic of radiolysis related substances.

After dissolution in Milli Q water, irradiated samples of ampicillin give UV spectra reported in Fig. 7. In the region of high absorbance, dilute solutions $(0.1 \%)$ are necessary to distinguish the maxima at 255, 260 and $270 \mathrm{~nm}$, concentrated solutions (1\%) are used to study the $335 \mathrm{~nm}$ maxima characteristic of the related substances.

As expected, the absorbances at 287 and $320 \mathrm{~nm}$ are function of the radiation dose (Fig. 8). However it will be noticed that the absorbances at 255, 260 and $270 \mathrm{~nm}$ also increase with the absorbed radiation dose; the increase is slower than that at the previous wavelengths, but significant.

In order to study the change in the total UV spectra with irradiation, we use the ratio of the spectra; the recorded spectrum after one radiation dose is divided by the reference spectrum without radiolysis. That is the limit of the method, a reference spectrum $(A \neq 0)$ is necessary as observed in several tests with others molecules. In this study, the values of the ratio progressively increase with the radiation dose (Fig. 9).

The ratio-dose relationship for the two wavelengths of maximum absorbance is given in figure 10 . The ratio increases linearly with radiation dose, the best fit line is given by $\mathrm{y}=0.17 \mathrm{x}+0.98$ at $287 \mathrm{~nm}\left(\mathrm{R}^{2}=0.998\right)$. 


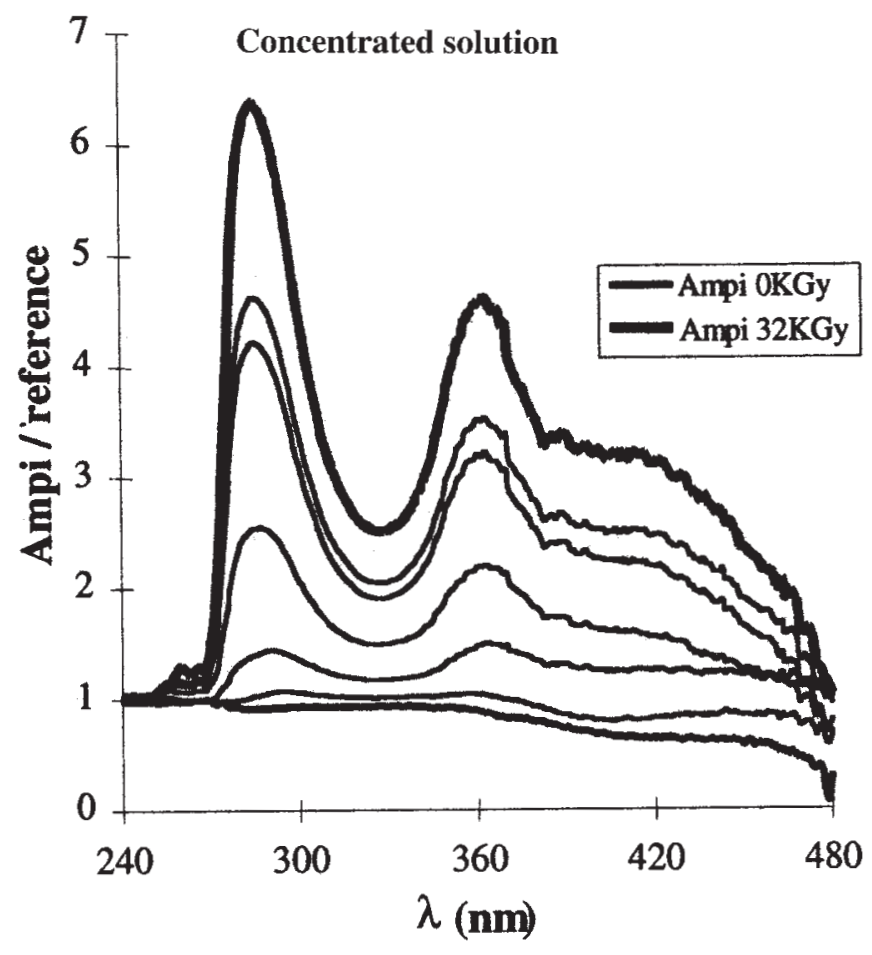

Fig. 9. Increase of the ratio of spectra from ampicillin at increasing dose and the spectrum of nonirradiated ampicillin (reference). The doses are $0 ; 0.23 ; 0.9 ; 2.3 ; 8.8 ; 19 ; 21$ and $32 \mathrm{kGy}$ respectively.

Similar results are obtained with samples irradiated in solid state and kept at $-20^{\circ} \mathrm{C}$ for 9 months before dissolution in Milli Q water. However, the ratio-dose relationship is different at $287 \mathrm{~nm}$ : $\mathrm{y}=0.28 \mathrm{x}+1.43$. These results are similar but amplified. The ratio value at this wavelength is 10 instead of 6.5 immediately after the irradiation.

\section{Discussion}

\section{Dose effect}

HPLC with UV spectroscopy analysis allows unambiguous detection of radiosterilization for ampicillin: new peaks are produced in the chromatogram (unique) and the shape of the related substances is quite different (Fig. 1 and Fig. 2). However it is difficult to give a general quantitative relationship, with increasing radiation dose of irradiation, some peaks are reduced (peak $22 \mathrm{~min}$ ), others increase, either linearly (peak $28 \mathrm{~min}$ ) or towards a plateau value (peaks 20 and $26 \mathrm{~min}$ ) (Fig. 3).

For the accumulation of trapped radicals, the yields start to be reduced from 15 to $25 \mathrm{kGy}(5,6)$. The effects on yields of peaks 20 and 26 are similar as expected; the radicals are the intermediate in the production of final products.

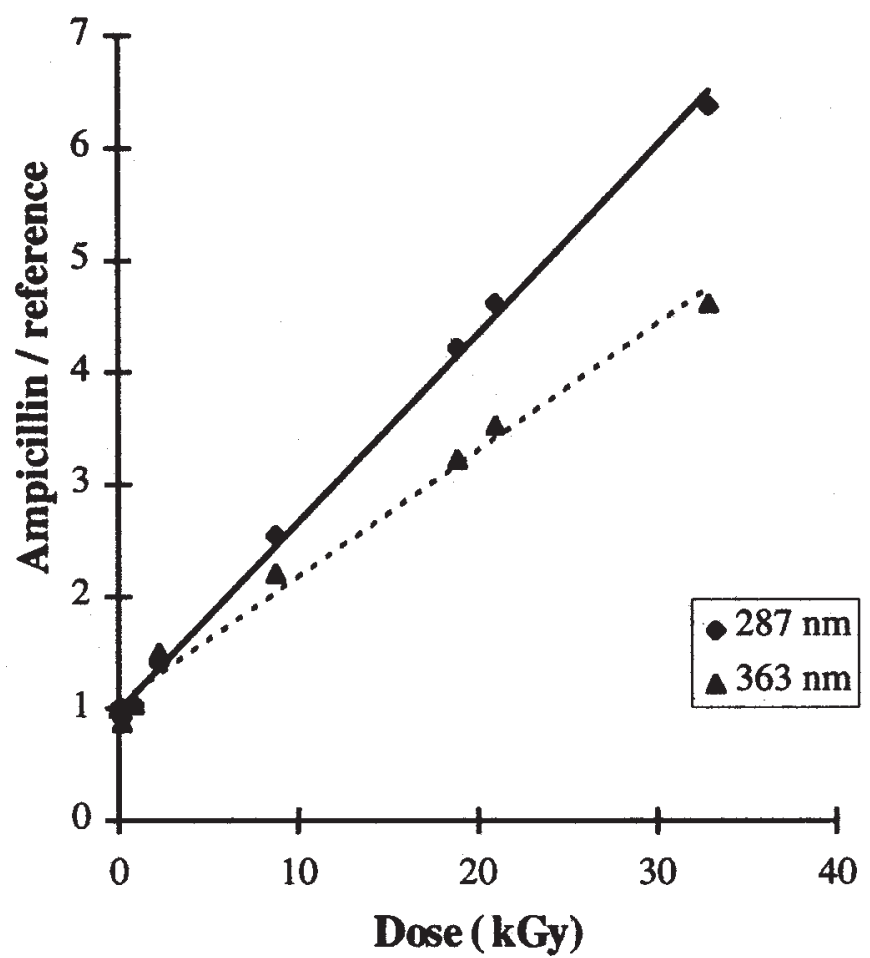

Fig. 10. Spectra ratios registered at 287 and $363 \mathrm{~nm}$ as a function of the dose (sec Fig. 9 for all wavelengths).

The reduction of the peak 22 is also predictable. In solid state, the energy migrates along the matrix and a part may be transferred into selected defects as impurities already present in related substances before the radiolysis. The energy transfer (electron tunneling, electronic excitation energy) excites the molecule of impurity and during the relaxation, the molecule is transformed in final product. An other hypothesis is a direct radiolytic effect on peak 22 .

Some products such as peak $28 \mathrm{~min}$ are unique to the radiolysis and are produced by radiolytic mechanisms. The radical contribution to their production must be negligible, the accumulation curve is not of the radical type.

The "dose effect" is different for each peak but must be explained by the accumulation in the solid of radiolytic products with different capacity to scavenge particle or energy.

\section{Potency}

The potency is not affected significantly by the radiosterilization. In the solid state, the energy diffuses into the solid and the yields are lower than those in aqueous solution; a yield of $10^{-7}$ mole $\mathrm{J}^{-1}$ is typical for a single product and correspond to $\left(10^{-7} \mathrm{~mole} / \mathrm{J}^{-1}\right.$ x $\left.25,000 \mathrm{~J} \mathrm{~kg}^{-1}\right) 2.510^{-3} \mathrm{~mole} / \mathrm{kg}$ or, for ampicillin, $0.9 \mathrm{~g}$ of products per $\mathrm{kg}$. The experimental decrease of ca. $4 \%$ (40 $\mathrm{g}$ of products per $\mathrm{kg}$ ) is a proof 


\section{Original articles}

of the great number of final products. As a rule, radiolytic products are numerous but in low quantities. The concentration is too low to obtain any response in toxicity tests (7).

\section{Spectra}

In figure 5, the comparison between capillary HPLC and classical HPLC shows that the capillary system exhibits more details and, for the same injected quantity give sharper and higher peaks and so, more information. Retention times are almost similar but a little shorter in the conventional than in the capillary column. The spectra (Fig. 6) of radiolytic compounds (peaks 20-26) are quite similar but are very different from the impurity spectrum of unirradiated ampicillin (usual related compounds). The most remarkable features are maxima at 287 and $335 \mathrm{~nm}$ characteristic of the radiolytic compounds. So the question is: is it possible to use this range of wavelengths with an usual UV spectrophotometer to detect a signal after the radiolysis?

The answer is affirmative. With a concentrated solution of ampicillin irradiated in solid state at 33,000 Gy an absorbance appears in the $300-350 \mathrm{~nm}$ range (Fig. 7). This absorbance is function of the radiation dose (Fig. 8), so this signal can be used to detect the irradiation. In dilute solution, we concentrate on the $250-270 \mathrm{~nm}$ range but the variation of the absorbance with the radiation dose is limited and may be difficult to distinguish by a rapid study, particularly at low radiation doses (Fig. 8). By contrast, using the ratio of the experimental spectra (Fig. 9), it will be easy to detect the irradiation for radiation doses as low as 3,300 Gy. With $10 \mathrm{kGy}$, the ratio is very informative for the 287 and $363 \mathrm{~nm}$ wavelengths (Fig.10). This conclusion is reinforced if we look at Fig. 10 where the effect of the radiation dose is given for both wavelengths, and is seen to increase linearly with radiation dose.

During the storage of the products in excellent conditions (low temperature, closed samples, protected from light), the detection becomes more and more easy proving that slow mechanisms are still possible and occur leading to more products. It is well known that, after the radiolysis, the yield of trapped radicals is very slowly reduced, the radical decay ( -40 to $-60 \%)$ takes several months (1). So, we conclude that trapped radicals give "coloured" compounds during the radiolysis (the radiation dose effect is similar) and after the radiolysis (radicals decay and radiolytic product peaks increase after the radiolysis).

\section{Conclusion}

For ampicillin samples, radiosterilization may be detected by an usual UV-spectrometer. In this method, the problem is that the reference spectrum already present an absorbance, because values from this spectrum is used to divided values from the experimental spectrum.

\section{References}

1. Barbarin, N.; Crucq, A.-S.; Tilquin, B. Rad. Phys. Chem. 1996, 48, 787-794.

2. Gibella, M.; Crucq, A.-S.; Tilquin, B. J. Chim. Phys. 1993, 90, 1041-1053.

3. Gibella, M. Selection de méthodes analytiques pour la détection de la radiostérilisation. Ph. D. Thesis UCL (Université Catholique de Louvain). Louvain-La-Neuve, 1998.

4. Gibella, M.; Crucq, A.-S.; Tilquin, B.; Stocker, P.; Lesgards, G.; Raffi, J. Electron spin resonance studies of some irradiated pharmaceuticals. (submitted to publication in Radiation Physics and Chemistry).

5. Jacobs,G.P. Rad. Phys. Chem. 1985, 26, 133-142.

6. Sanderson, D.C. Luminescence detection of irradiated foods in Food Irradiation and the Chemist in Royal Society of Chemistry ( ${ }^{\circ}$ 86). Edited by: Johnston \& Stevenen, 1990.

7. Zeegers, F.; Gibella, M.; Tilquin, B. Rad. Phys. Chem. 1997, 50, 149-151. 